\title{
A good marker does not mean a good target for clinical trials in Alzheimer's disease: the amyloid hypothesis questioned
}

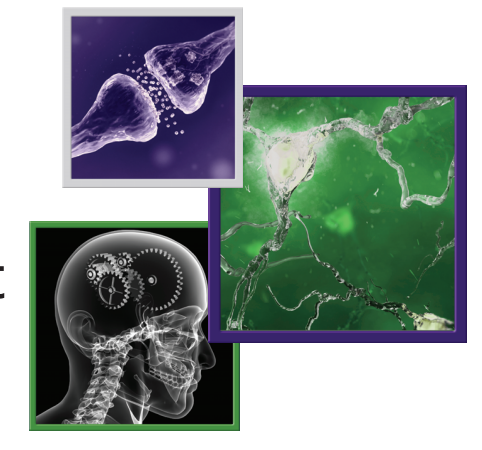

\author{
Pedro Modrego*,1 \& Antonio Lobo ${ }^{2}$ (iD) \\ ${ }^{1}$ Department of Neurology, Hospital Universitario Miguel Servet, Aragon, Spain \\ ${ }^{2}$ Department of Psychiatry, University of Zaragoza, Aragon, Spain \\ *Author for correspondence: pmodrego@salud.aragon.es
}

\begin{abstract}
" more research in basic science is essential to untangle the pathogenesis of AD. Alternative hypotheses deserve more attention, even those pointing to infective agents, for which there is some evidence with regard to infectious components, causation and mechanisms"
\end{abstract}

First draft submitted: 15 April 2019; Accepted for publication: 26 April 2019; Published online: 22 May 2019

Keywords: Alzheimer's disease $\bullet$ amyloid hypothesis $\bullet$ clinical trials

Many clinical trials have been conducted in Alzheimer's disease (AD) patients, mainly based on anti-amyloid drugs. However, the results have not yielded positive results. Ongoing trials with anti-amyloid and anti-tau agents are expected to terminate in the forthcoming years. There are several possible reasons for trial failures: an inadequate target, treatment in an advanced stage of disease and the heterogeneity of AD in terms of the variety of protein system dysfunctions. Future trials should be based on the development of a new, comprehensive hypothesis explaining the connection and interactions of the abnormal proteins. This editorial discusses the challenges encountered to date, and requirements for future research.

$\mathrm{AD}$ is one of the main priorities of healthcare systems in western countries, given the high prevalence in an aging population. In the last two decades, important developments have been made in the field of biomarkers in AD. However, currently available treatments for $\mathrm{AD}$ are disappointing. With 900 interventional studies completed and registered in the ClinicalTrials. gov database, only five drugs have been approved by the US FDA and marketed in western countries: tacrine, donepezil, rivastigmine, galantamine and memantine.

The cholinergic hypothesis has been of limited value with regard to trial design, and is now exhausted. Most recent trials have relied on the amyloid hypothesis, with the target of $\beta$-amyloid protein. This protein is the main component of the amyloid plaque, which is the hallmark of $\mathrm{AD}$. While it is true that amyloid deposition is always seen in $\mathrm{AD}$ cases, its pathogenicity has been questioned, with amyloid protein representing rather an end-stage of the disease than a cause [1]. In addition, the connection between amyloid protein and tau pathology is far from being understood. Given that all clinical trials conducted to date with anti-amyloid drugs have failed to demonstrate efficacy on end points (Table 1), many researchers have raised alternative causal hypotheses. When repeated trials focused on the same target fail, questions arise as to whether this is the appropriate target. The amyloid hypothesis has outweighed all other etiological hypotheses, resulting in lost opportunities.

Apart from etiological considerations, $\mathrm{AD}$ is a more heterogeneous condition than previously thought. A systematic review of cohort studies showed that there is a wide variability in clinical symptoms, survival and clinical progression among $\mathrm{AD}$ patients [2]. The same is also true at the level of proteomics. A total of $353 \mathrm{AD}$ patients were classified into five subgroups by clinical profile, based on APOE genotype, age, and CSF levels of amyloid $\beta 42$ $(\mathrm{A} \beta-42)$, tau and ubiquitin proteins, with important interactions among them [3]. Neuropathological studies also point to important heterogeneity. Significant differences in the protein composition of senile plaques were found in a series of $44 \mathrm{AD}$ patients. A total of 913 proteins were identified on average in plaques and 279 found in every patient. Patients with faster disease progression had more neuronal synaptic proteins and fewer glial proteins [4]. In another series of $247 \mathrm{AD}$ patients, co-pathology of $\alpha$-synuclein and TDP- 43 was frequently found, and the 
Table 1. Anti-amyloid drugs that failed to show clinical improvement in Alzheimer's disease.

\begin{tabular}{|ll|}
\hline Drug type & Drug \\
\hline Monoclonal antibody & Bapineuzumab in Phase III trials \\
\hline & Solaneuzumab in Phase III trials \\
\hline Gamma secretase inhibitor & Crenezumab in Phase II trials \\
\hline & Gantenerumab in Phase III trials \\
\hline Beta secretase inhibitor & Avagacestat in Phase II trials \\
\hline & Semagacestat in Phase III trials \\
\hline & Verubecestat in a Phase III trial \\
\hline & Lanabecestat in Phase III trials \\
\hline
\end{tabular}

same was seen in other neurodegenerative diseases such as frontotemporal dementia, Lewy body disease, progressive supranuclear paralysis and corticobasal degeneration [5]. Furthermore, in a series of 889 AD cases studied with neuropathology, three subtypes were identified: typical, limbic predominant and hippocampal sparing. Important differences were found with regard to neurofibrilary tangles, APOE genotype, atrophy and age of presentation [6].

Active (AN1792, ACC-001, CAD106, AFFITOPE) and passive immunizations have not yielded positive results in humans [7], but we have learned something from this research. Among the 64 patients vaccinated in the AN1792 trial, 34 subsequently died, making necropsy possible; and the brain was examined in nine cases. In two patients, plaque clearance was minimal; there was intermediate plaque removal in four cases; and complete removal in two. However, no clinical improvement was seen in any of the patients examined [8].

An interim analysis of a randomized clinical trial $(n=165)$ with aducanumab has also shown a decrease in amyloid plaques in the brain in a dose- and time-dependent manner, in comparison with placebo, and to slow cognitive decline [9]. Two Phase III clinical trials are underway, which aim to recruit more than 1300 patients each $[10,11]$. Both are expected to conclude in 2022 . There are also ongoing trials with anti-tau therapies, although others have been discontinued because of toxicity concerns. The results of these trials will either support or weaken the amyloid hypothesis.

Assuming that $\mathrm{AD}$ is essentially a proteinopathy, $\beta$-amyloid and tau protein accumulation are not the only pathogenic agents, with complex interactions with other proteins and other agents. Recent advances in proteomics have shown that there is a complex map of protein alterations across brain areas in $\mathrm{AD}$, with many proteins increased and other decreased [12]. It suggests multiple system failure in various aspects of protein metabolism, such as production, transport clearance and degradation by autophagy. Therefore, conducting clinical trials with antiamyloid drugs may result in the waste of time and resources. Conversely, more research in basic science is essential to untangle the pathogenesis of $\mathrm{AD}$. Alternative hypotheses deserve more attention, even those pointing to infective agents, for which there is some evidence with regard to infectious components, causation and mechanisms [13].

Alternative/additional reasons, apart from an inadequate target, have been proposed to explain the failure of clinical trials: insufficient number to treat, insufficient trial duration, drugs given too late in disease, inadequate dosing and problems with rating tools. However, future clinical trials will need better target definition [14].

A comprehensive approach is needed for drug development in $\mathrm{AD}$, and ideally, new drugs should be able to act in both amyloid deposition and tau formation. New developments in epigenetics and pharmacoepigenomics may pave the way for effective drugs. In this respect, DNA hypomethylation is associated with the progression of $\mathrm{AD}$, so methylation promoters could decrease expression of genes involved in amyloid and tau deposition [15]. Recently, a nonselective histone deacetylase inhibitor (HDAC3 inhibitor/silencing) has demonstrated the following effects in transgenic mice: decreased tau phosphorylation/acetylation, reduced $\beta$-secretase cleavage, decreased A $\beta$ 42 accumulation and spatial memory improvement. Furthermore, repeated administration of the drug reversed tau phosphorylation in neurons [16].

An epigenetic Drug Target Network has been constructed and 14 epigenetic repositioning drugs have been proposed on the basis of the different target proteins and their relation with noncoding RNAs [17].

Other potential treatments for $\mathrm{AD}$ include those based on the inhibition of glycogen synthase kynase 3 via mammalian target of rapamycin (mTOR) to promote $\beta$-amyloid clearance and decrease tau phosphorylation [18]. mTOR inhibition activates the ubiquitin proteasome system and autophagy [19]. Therefore, there is a glimmer of hope in the pipeline of drugs for $\mathrm{AD}$ with new mechanisms of action. 
Financial \& competing interests disclosure

The authors have no relevant affiliations or financial involvement with any organization or entity with a financial interest in or financial conflict with the subject matter or materials discussed in the manuscript. This includes employment, consultancies, honoraria, stock ownership or options, expert testimony, grants or patents received or pending, or royalties.

No writing assistance was utilized in the production of this manuscript.

\section{References}

1. Castellani RJ, Lee HD, Zhu X, Nunomura A, Perry G, Smith MA. Neuropathology of Alzheimer disease: pathognomonic but not pathogenic. Acta Neuropathol. 111, 503-509 (2006).

2. Modrego PJ, Lobo A. Determinants of mortality and progression in Alzheimer's disease. A systematic review. Neuropsychiatry (London) 8(5), 1465-1475 (2018).

3. Iqbal K, Flory M, Khatoon S et al. Subgroups of Alzheimer's disease based on cerebrospinal fluid molecular markers. Ann. Neurol. 58, 748-752 (2005).

4. Drummond E, Nayak S, Faustin A et al. Proteomic differences in amyloid plaques in rapidly progressive and sporadic Alzheimer's disease. Acta Neuropathol. 133, 933-954 (2017).

5. Robinson JL, Lee EB, Sie SX et al. Neurodegenerative disease concomitant proteinopathies are prevalent, age-related, and APOE4-associated. Brain 141, 2181-2193 (2018).

6. Murray ME, Graff-Radford NR, Ross OA, Petersen RC, Duara R, Dickson DW. Neuropathologically defined subtypes of Alzheimer's disease with distinct clinical characteristics: a retrospective study. Lancet Neurol. 10, 785-796 (2011).

7. Yang C, Xiao S. New development of clinical trial in immunotherapy for Alzheimer's disease. Curr. Pharm. Biotechnol. 16, 484-491 (2015).

8. Holmes C, Boche D, Wilkinson D et al. Long-term effects of Abeta 42 immunisation in Alzheimer's disease: follow-up of a randomised, placebo-controlled Phase I trial. Lancet 372, 216-223 (2008).

9. Sevigny J, Chiao P, Bussière T et al. The antibody aducanumab reduces A plaques in Alzheimer's disease. Nature 537, 50-56 (2016).

10. US National Institutes of Health. https://clinicaltrials.gov/ct2/show/NCT02477800

11. US National Institutes of Health. https://clinicaltrials.gov/ct2/show/NCT02484547

12. Li KW, Ganz AB, Smit AB. Proteomics of degenerative diseases: analysis of human postmortem brain. J. Neurochem. doi:10.1111/jnc. 14603 (2018) (Epub ahead of print).

13. Itzhaki RF, Lathe R, Balin BJ et al. Microbes and Alzheimer's disease. J. Alzheimers Dis. 51, 979-984 (2016).

14. Mehta D, Jackson R, Paul G, Shi J, Sabbagh M. Why do trials for Alzheimer's disease drugs keep failing? A discontinued drug perspective for 2010-2015. Expert Opin. Investig. Drugs 26, 735-739 (2017).

15. Tejido O, Cacabelos R. Pharmacoepigenomic interventions as novel potential treatments for Alzheimer's and Parkinson's diseases. Int. J. Mol. Sci. 19, 3199 (2018).

16. Janczura K, Volmar CH, Sartor GC et al. Inhibition of HDAC3 reverses Alzheimer's disease-related pathologies in vitro and in the 3xTg-AD mouse model. Proc. Natl Acad. Sci. USA 115, E11148-E11157 (2018).

17. Chatterjee P, Roy D, Rathi N. Epigenetic drug repositioning for Alzheimer's disease based on epigenetic targets in human interactome. J. Alzheimers Dis. 61, 53-65 (2018).

18. Zhu Y, Wang J. Wagonin increases $\beta$-amyloid clearance and inhibits tau phosphorylation via inhibition of mammalian target of rapamycin: potential drug to treat Alzheimer's disease. Neurol. Sci. 36, 1181-1188 (2015).

19. Zhao J, Zhai B, Gygi SP, Goldberg AL. mTOR inhibition activates overall protein degradation by the ubiquitin proteasome system as well as by autophagy. Proc. Natl Acad. Sci USA 112, 15790-15797 (2015). 
